Stabile Angina pectoris

\title{
Beschwerden oft durch mikrovaskuläre Störungen verursacht
}

\begin{abstract}
Bei den meisten Patienten mit Angina pectoris werden bei der Angiografie keine hämodynamisch relevanten Koronarstenosen gefunden. Auslöser der Beschwerden sind vielmehr mikrovaskuläre Störungen, die am besten konservativ behandelt werden.
\end{abstract}

Laut Registerdaten aus den USA wird nur bei rund $40 \%$ der Patienten mit Verdacht auf KHK bei einer Herzkatheteruntersuchung eine obstruktive KHK nachgewiesen. Bestätigt wird dies durch Daten einer aktuellen deutschen Studie bei 300 Patienten mit positivem Ischämietest, berichtete Priv.-Doz. Dr. med. Dirk Westermann, Kardiologe an der Charité in Berlin. Nur bei $46 \%$ lag eine obstruktive KHK vor (Stenosegrad > 50\%), bei $47 \%$ war das Angiogramm normal (Stenosegrad 0-20\%). Die pektanginösen Beschwerden wurden bei diesen Patienten häufig durch epikardiale oder mikrovaskuläre Spasmen ausgelöst. Diese Ursachen hängen auch mit lonenkanalstörungen und einem verstärkten späten $\mathrm{Na}^{+}$-Strom in die Herzmuskelzelle zusammen, sagte Westermann.
Zusätzlich zu einer hämodynamisch guten Einstellung der Patienten (Blutdruck und Herzfrequenz im Zielbereich) ist bei persistierenden Beschwerden eine Behandlung mit Ranolazin sinnvoll, das direkt an der Herzmuskelzelle angreift und den späten $\mathrm{Na}^{+}-$ Einstrom hemmt. Durch die zusätzliche Gabe von Ranolazin (Ranexa ${ }^{\oplus}$ ) können die Zeit bis zum Auftreten einer erneuten Ischämie mit pektanginösen Beschwerden sowie die Belastungsdauer verlängert werden, berichtete Priv.-Doz. Dr. med. Samuel T. Sossalla aus Göttingen. In der Zulassungsstudie zu Ranolazin war zudem unter der Therapie die Häufigkeit ventrikulärer Tachykardien fast halbiert (5,3 vs. 8,3\% Placebo). Die Verbesserung der Koronarperfusion durch Ranolazin bei Patienten mit wiederkehrenden Symptomen einer Angina pectoris (AP), die keine obstruktive KHK hatten, ist bereits in einer placebokontrollierten Crossover-Studie bei 20 Frauen belegt worden.

(rf) II

II Symposium, DGK-Herbsttagung, Hamburg, 12. Oktober 2012 (Veranstalter: BerlinChemie)

\section{Antikoagulation bei Vorhofflimmern}

\section{Bessere Schlaganfallprophylaxe nötig}

Die Sterblichkeit von Patienten mit Vorhofflimmern ist um das Zwei- bis Dreifache im Vergleich zur nicht erkrankten Altersgruppe erhöht. Die Antikoagulation reduziert das Schlaganfallrisiko - sofern sie richtig angewendet wird.

Derzeit befinden sich die antikoagulierten Patienten mit Vorhofflimmern nur zu etwa $69 \%$ der Zeit innerhalb der therapeutischen Zielbreite, erklärte Prof. Christoph Bode aus Freiburg. Innerhalb des INR-Bereichs von 2,0-3,0 besteht ein Schutz vor Schlaganfall, darüber steigt das Blutungsrisiko. Als Alternative zum Vitamin-K-Antagonisten Phenprocuomon (Marcumar ${ }^{\circledR}$ ) bietet sich das neue Antikoagulans Rivaroxaban (Xarelto ${ }^{\circledR}$ ) an, wie Bode ausführte. Die ROCKET-HF Studie hatte ergeben, dass Rivaroxaban (20 mg einmal täglich) im Vergleich zu Warfarin (INR 2,0-3,0) in der Wirksamkeit nicht unterlegen ist. Zudem kam es unter Rivaroxaban zu signifikant weniger schweren Blutungen als unter Warfarin.

Inzwischen ist Rivaroxaban zur Prävention von Schlaganfällen und systemischen Embolien bei erwachsenen Patienten mit nicht valvulärem Vorhofflimmern in mehr als 60 Ländern zugelassen, darunter Europa und die USA.

(rk) II

II Expertengespräch, ESC-Kongress, München, 24. August 2012 (Veranstalter: Bayer)
Kurz notiert

Online-Fortbildung

"Kardiale Ischämie"

Haben Patienten Beschwerden einer Mangeldurchblutung im Herzen, kommen vom Kardiologen aber ohne interventionspflichtigen Befund wieder, stellt sich die Frage, was zu tun ist. "Da ist keine Stenose", sagt der Kardiologe, ,ich habe Beschwerden", der Patient.

Was steckt hinter einer wiederkehrenden kardialen Ischämie, was ist der Pathomechanismus, welche therapeutischen Optionen gibt es, welche antiischämischen Wirkstoffe sind verfügbar, wie wirkt Ranolazin? Antworten bietet in rund zehn Minuten die Online-Fortbildung "kardiale Ischämie" von BerlinChemie. Sie ist zu finden unter www.ranexa-schulung.de.

In Berlin-Chemie

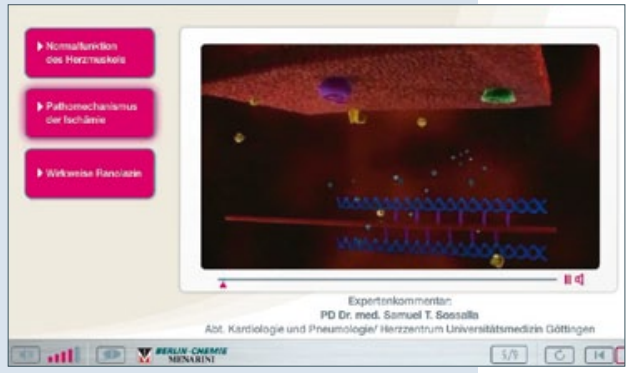

Nitrolingual ${ }^{\circledR}$

Forschungspreis 2013

Um den wissenschaftlichen Dialog um Glyceroltrinitrat zu fördern, lobt Pohl-Boskamp den internationalen Nitrolingual ${ }^{\circledR}$-Forschungspreis 2013 aus, der mit 10000 Euro dotiert ist. Bewerben können sich Nachwuchswissenschaftler der Fachbereiche Medizin und Naturwissenschaften mit bisher unveröffentlichten Arbeiten über neue pharmakologische und klinische Erkenntnisse zu Glyceroltrinitrat. Einsendeschluss für die Manuskripte ist der 1. Mai 2013. Alle Details zur Auslobung sind zu finden unter www. pohlboskamp.de.

II Pohl-Boskamp 